\title{
Physiologically based pharmacokinetic modeling for predicting irinotecan exposure in human body
}

\author{
Yingfang Fan ${ }^{1,2, *}$, Najia Mansoor ${ }^{3, *}$, Tasneem Ahmad4, Rafeeq Alam Khan ${ }^{3}$, Martin \\ Czejka $^{5}$, Syed Sharib ${ }^{4}$, Dong-Hua Yang ${ }^{2}$ and Mansoor Ahmed ${ }^{6}$ \\ ${ }^{1}$ Department of Hepatobiliary Surgery, Zhujiang Hospital, Southern Medical University, Guangzhou 510280, China \\ ${ }^{2}$ Department Pharmaceutical Sciences, College of Pharmacy and Health Sciences, St. John's University, Queens, NY 11439, USA \\ ${ }^{3}$ Department of Pharmacology, Faculty of Pharmacy \& Pharmaceutical Sciences, University of Karachi, Karachi 75270, Pakistan \\ ${ }^{4}$ Pharma Professional Services, Karachi 75270, Pakistan \\ ${ }^{5}$ Department of Clinical Pharmacy and Diagnostics, University of Vienna, A-1090 Vienna, Austria \\ ${ }^{6}$ Department of Pharmaceutical Chemistry, Faculty of Pharmacy \& Pharmaceutical Sciences, University of Karachi, Karachi \\ 75270, Pakistan \\ *These authors have contributed equally to this work \\ Correspondence to: Mansoor Ahmed, email: cando@cyber.net.pk \\ Dong-Hua Yang, email: yangd $1 @$ stjohns.edu \\ Keywords: physiologically based pharmacokinetics (PBPK), irinotecan, colorectal cancer \\ Received: March 24, $2017 \quad$ Accepted: May 04, $2017 \quad$ Published: June 06, 2017 \\ Copyright: Fan et al. This is an open-access article distributed under the terms of the Creative Commons Attribution License 3.0 (CC BY 3.0), \\ which permits unrestricted use, distribution, and reproduction in any medium, provided the original author and source are credited.
}

\section{ABSTRACT}

Colorectal cancer is the third leading cause of cancer-related deaths in the United States. Treatment of colorectal cancer remains a challenge to clinicians as well as drug developers. Irinotecan, a Camptothecin derivative, is successfully used for the treatment of this rapidly progressing malignancy and finds its place in the first line of therapeutic agents. Irinotecan is also effective in treating SCLC, malignant glioma and pancreatic adenocarcinoma. However, its adverse effects limit its clinical application. Mainly metabolized by hepatic route, and excreted through biliary tract, this dug has been found to possess high variation in patients in its pharmacokinetic (PK) profile. Physiologically based pharmacokinetic (PBPK) models using compartmental approach have attained their position to foresee the possible PK behavior of different drugs before their administration to patients and such models have been proposed for several anticancer agents. In this work, we used WB-PBPK technology to develop a model in a population of tumor patients who used IV irinotecan therapy. This model depicted the concentration of drug and its pharmacologically active metabolite in human body over a specific period of time. Knowledge about pharmacokinetic parameters is extracted from this profile and the model is evaluated by the observed results of clinical study presented in literature. The predicted behavior of the drug by this approach is in good agreement with the observed results and can aid in further exploration of PK of irinotecan in cancer patients, especially in those concomitantly suffer from other morbidity.

\section{INTRODUCTION}

Pharmacokinetic (PK) evaluation of anticancer agents is sparsely available in literature because of the highly inter individual variability exhibited by drugs. Anticancer drugs frequently show a narrow therapeutic index (TI), a fact that requires precise dosing in order to minimize toxicity while at the same time maintaining sufficient drug delivery to tumor cells needed for effective clinical activity. In recent years, physiologically based pharmacokinetic (PBPK) approaches have been successfully applied for the use of anticancer agents. PBPK modeling is based on an approach which starts with model building required both in vitro and in vivo data 
followed by model verification. Various PBPK models have been successfully used for some small molecular targeting drugs in predicting their exposure to human body and designing phase I clinical studies [1].

Early from the start of this century, the development of different models using PBPK modeling and simulation technique to describe detailed PK/PD data of multiple drugs is increasingly gaining attention from all aspects including academia, industry, and regulatory authorities and has now been established as an advanced approach for drug exposure-response analysis and clinical trial simulations $[2,3]$.

PBPK modeling and simulation technique possesses a huge potential and aids in efficient mechanistic understanding of pharmacokinetic as well as pharmacodynamic behavior of a drug and its metabolites. This understanding enables therapists to confidently make decisions regarding therapeutic strategies about clinical scenarios that have not yet been tested experimentally. Most importantly conducting population studies using PBPK approaches provides data that can be used to save time and resources otherwise required for experimental studies and to make experimental clinical trials confirmatory rather than exploratory.

In clinical practice, the required therapeutic doses of almost all anticancer agents are calculated on the basis of body surface area (BSA). However, it has been reported that the PK of many anticancer agents are not necessarily related to BSA [4]. Although the value of BSA-based dosing is frequently questioned [5], this approach is commonly practiced [6]. To identify the patient factors that involve in predicting drug exposure and its ultimate pharmacodynamic response, population-based evaluations via simulation approach are carried out [7], that are extremely useful in the development of appropriate dosing regimens. In 1998, PK and PK-PD model implementation had been suggested to revise the dosage of chemotherapeutic agents after measurement of drug concentration [8]. In the following decade, Evans et al. [9] reported that the 5-year survival in children with acute B-cell lymphoblastic leukemia improved from $66 \%$ to $76 \%$ by individualizing the dose of methotrexate based on individual patient's ability to clear the drug from plasma. A fully individualized methotrexate dosing in pediatric patients has also been suggested by other researchers further improving treatment outcomes [10]. Although a prospective clinical trial fully powered by modeling and simulation approach evaluating the outcomes predicted by this technique compared with standard care dosing system still awaits to be carried out, there are high expectations that this approach would be useful in near future to reduce toxicity while maintaining or even in some cases improving the treatment efficacy.

Usually, PK models are mainly based on mathematical mass balance equations that characterize drug absorption and disposition within the body. The PBPK models also consider anatomical and physiological realities, taking into account the various differences of distribution within and among organs in conjunction with their varying blood flows $[11,12]$. A typical whole -body PBPK approach conceptualizes multiple compartments representing different organs of the body linked together by the arterial and venous routes of blood circulation.

Whole-body PBPK models have been reported in literature for several clinically used antineoplastic agents, such as methotrexate, 5-fluorouracil, adriamycin, doxorubicin, and for many newer compounds demonstrating antitumor activities such as everolimus and genistein $[13,14]$. These models indicate how PBPK models can be useful in representing mechanistic drug-dependent features ultimately leading to more accurate predictions and in providing insights on physiological variables that can influence a drug disposition and whether concomitantly given drugs might interact with each other effecting the final outcome of the therapy.

Colorectal cancer is among one of the most common and deadly malignancies with an incidence of 1.2 million new cases per year globally and is estimated to be the third most common cause of cancer-related deaths in USA [15]. It has been considered as moderately resistant to chemotherapy. Though colonoscopic screening has considerably reduced the death rate of this disease in developed countries, patients in most under developed and developing countries are still diagnosed at advanced stages due to the expenses of colonoscopy added with poor access to health care.

Irinotecan (CPT-11) is a camptothecin derivative which is metabolized to produce 7-ethyl-10-hydroxycamptothecin (SN-38) after carboxyl esterase-mediated breakdown [16] (Figure 1). SN-38 is the active metabolite of irinotecan [17] that inhibits topoisomerase I, a nuclear enzyme that is essentially required for relaxation of super coiled DNA [18] and is $100-1000$ times more potent than Itinotecan, inducing cytotoxic changes and finally causing apoptotic death of the malignant cells $[19,20]$. Further inactivation and metabolism of SN-38 to SN-38G occurs through hepatic uridine diphosphate glucuronosyl transferase 1A1 (UGT1A1) [19].

CPT-11received FDA approval in 1998 for the treatment of metastatic colorectal carcinoma that has recurred or progressed following treatment with standard chemotherapy [21]. Avastin and CPT-11 combination therapy has shown rapid clinical as well as radiographic improvements in patients with relapsed malignant glioma. It has been reported that Topoisomerase I and II activities are significantly enhanced in malignant gliomas following DNA damage [22] by the single-agent irinotecan therapy. Irinotecan has also been shown to be effective in patients with extensive SCLC. New indication of Irinotecan has been reported in metastatic pancreatic ductal adenocarcinoma [23].

This paper presents the first detailed PBPK description of Irinotecan pharmacokinetics in human tumor patients by developing a PBPK model for irinotecan IV infusion of $350 \mathrm{mg}$ for 30 minutes. The model 
describes the pharmacokinetic parameters considering realistic human parameters, such as blood flow indifferent organs and the tissue/blood partition.

\section{RESULTS}

All results were depicted using standard Pk-Sim and MoBI graphical output. Numerical evaluation and visual predictive checks were adapted for comparison of predicted and clinically observed results. The graphical representation of plasma-concentration profile of CPT-11 and $\mathrm{SN}-38$ in comparison to the experimentally observed data in our generated population of patients was shown in Figure 2, The PK analysis of these compounds in terms of the mean values of parameters are shown in Table 1, which are AUC $1296.06 \mu \mathrm{mol} / \mathrm{min} / 1, \mathrm{C}_{\max } 9.86 \mu \mathrm{mol} / 1$. half life $29.21 \mathrm{~h}$ and the $\mathrm{t}_{\max } 1.5 \mathrm{~h}$. For irinotecan's active metabolite $\mathrm{SN}-38$, the mean AUC, $\mathrm{C}_{\max }, \mathrm{t}^{1 / 2}$ and $\mathrm{t}_{\max }$ values calculated for this population were $30.37 \mu \mathrm{mol} / \mathrm{min} / 1,0.07$ $\mu \mathrm{mol} / 1 ., 19.47 \mathrm{~h}$ and $1.7 \mathrm{~h}$ respectively. The prediction of pharmacokinetics was enabled due to the incorporation of different physico-chemical properties. Lipophilicity $(\log$ P), Fraction unbound of drug and PKa values have shown impact on the prediction of plasma concentration profile. Log P value determines the ability of drug to transport through membranes, Fraction unbound of drug lead to determination of volume of distribution of drug and PKa has a direct impact on the Lipophilicity and protein binding of the drug i.e. eventually related to drug pharmacokinetics [24]. The mean and $5^{\text {th }}$ to $95^{\text {th }}$ percentile profiles of CPT-11 and SN-38 are shown graphically in Figure 3. Figure 4 depicts the comparative plasma concentrations of $\mathrm{SN}-38$ and water soluble $\mathrm{SN}-38 \mathrm{G}$ versus time. It is evident from Figures 3 and 4 that SN38 is extensively metabolized by glucuronidated enzyme presenting greater concentration of SN-38G than SN-38 in plasma. Our physiologically based compartmental analysis method for predicting human PK yielded good predictive results and show that these pharmacokinetic profiles of irinotecan and $\mathrm{SN}-38$ were in agreement with the observed data. These results indicated the ability of the model to describe irinotecan and its metabolite exposure in adults after IV application. Changing the $\mathrm{pH}$ environment of tumor tissue or blood plasma exhibited no detectable effects on pharmacokinetic parameters of parent drug or its metabolite as no difference of any degree was noted for $\mathrm{AUC}_{\text {tend }}, \mathrm{C}_{\max }$, half life, plasma clearance, MRT, and volume of distribution of CPT-11 and SN-38 at different $\mathrm{pH}$ ranges of both blood plasma and tumor tissue.

\section{DISCUSSION}

Malignant tumors often progress rapidly. It is important to make decisions for drug dose selection, adjustment and combination of different anticancer agents in a short period of time. Various anticancer agents are known to exhibit narrow therapeutic index and high subject variability. Experimental studies in cancer patient population to investigate the pharmacokinetics of drugs during drug development or after clinical application demand long periods of time, because of many hurdles such as less number of patient participation, patients'
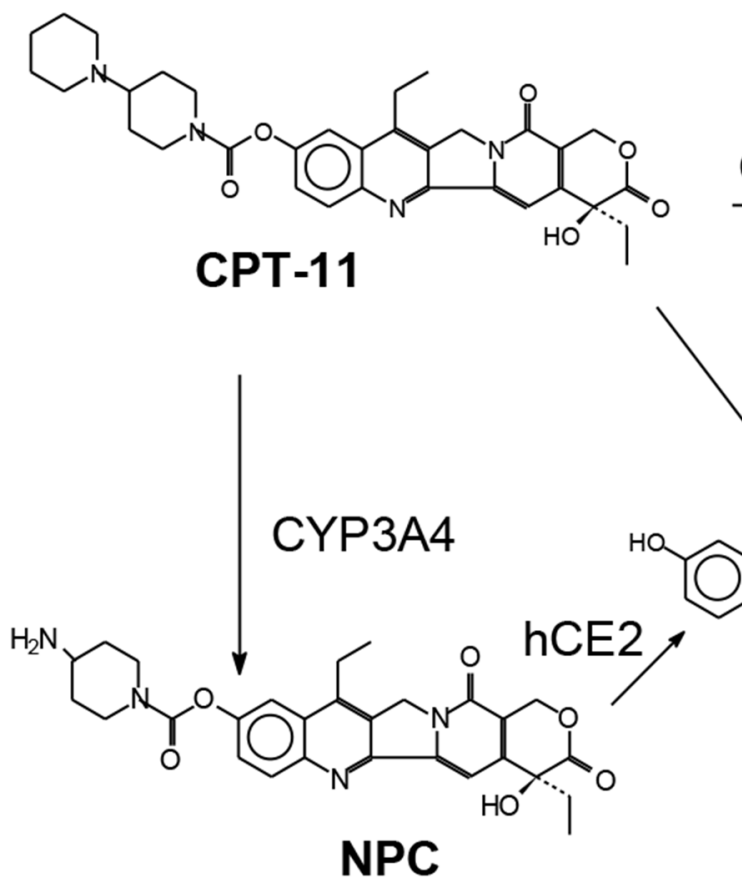

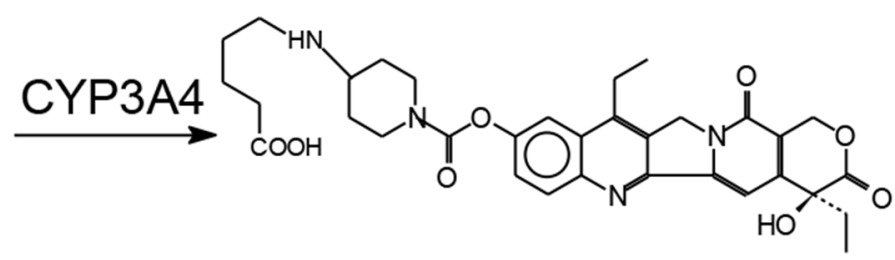

carboxyesterases

APC
SN-38

$<5 \%$

Figure 1: Chemical structure and metabolism of irinotecan. 
Table 1: Tumor population pharmacokinetics (100 individuals): $50 \%$ female and $50 \%$ male

Parameters

Concentration mean irinotecan plasma levels

AUC-tend $\left(\mu \mathrm{mol}^{*} \min / 1\right)$

AUC-inf $\left(\mu \mathrm{mol}^{*} \min / 1\right)$

AUC-inf-norm $(\mu \mathrm{g} * \min / 1)$

AUC- tend -norm $\left(\mu \mathrm{g}^{*} \mathrm{~min} / \mathrm{l}\right)$

$\operatorname{Cmax}(\mu \mathrm{mol} / 1)$

Cmax norm (mg/l)

C-tend ( $\mu \mathrm{mol} / 1)$

Total body clearance $(\mathrm{ml} / \mathrm{min} / \mathrm{kg})$

$\%$ AUC (t last- $\infty)$

MRT (h)

$\operatorname{tmax}(\mathrm{h})$

Half life (h)

Vdplasma (ml/kg)

Vdssplasma (ml/kg)
1296.06

1612.57

$\mathrm{NaN}$

$\mathrm{NaN}$

9.86

$\mathrm{NaN}$

0.13

$\mathrm{NaN}$

0.2

16.47

1.5

29.21

$\mathrm{NaN}$

$\mathrm{NaN}$
Concentration mean $\mathrm{SN}-38$ plasma levels

30.37

38.36

$\mathrm{NaN}$

$\mathrm{NaN}$

0.07

$\mathrm{NaN}$

0.00474

$\mathrm{NaN}$

0.21

16.45

1.7

19.47

$\mathrm{NaN}$

$\mathrm{NaN}$ frequent drop out from ongoing clinical research as a consequence of deteriorating general physical health owing to adverse effects of chemotherapeutic agents, and getting multiple blood samples in already debilitated patients with possible hematological abnormalities caused by chemotherapy, among others.

Modeling and simulation approaches make the envision of complex absorption, distribution, metabolism, and excretion outcomes of various drugs possible. These approaches can also predict profiles of parent drugs and their metabolite in various clinical scenarios. A growing interest in PBPK models is also the result of its utility in PK/PD-directed drug development [26, 27]. Simulations of virtual patient populations through these methods can save time and resources required by experimental studies and may play significant role in individualizing the dose of chemotherapeutic agents, increasing drug efficacy and desired consequences in every patient. These techniques also allow the simulation of virtual patient populations with altered physiological status such as obese/morbidly obese individuals and patients with renal impairment or liver cirrhosis.

Our present work is planned to provide a useful insight in the pharmacokinetic of IV infusion of Irinotecan in tumor patient population by the use of specialized physiologically based pharmacokinetic modeling technique. This work not only gives the plasma concentration profiles of parent drug irinotecan and its metabolite SN-38 but also provides their concentrations in tumor tissue which may provide opportunity for enhanced insight into pertinent PK thus aiding in design and adjustment of therapies.

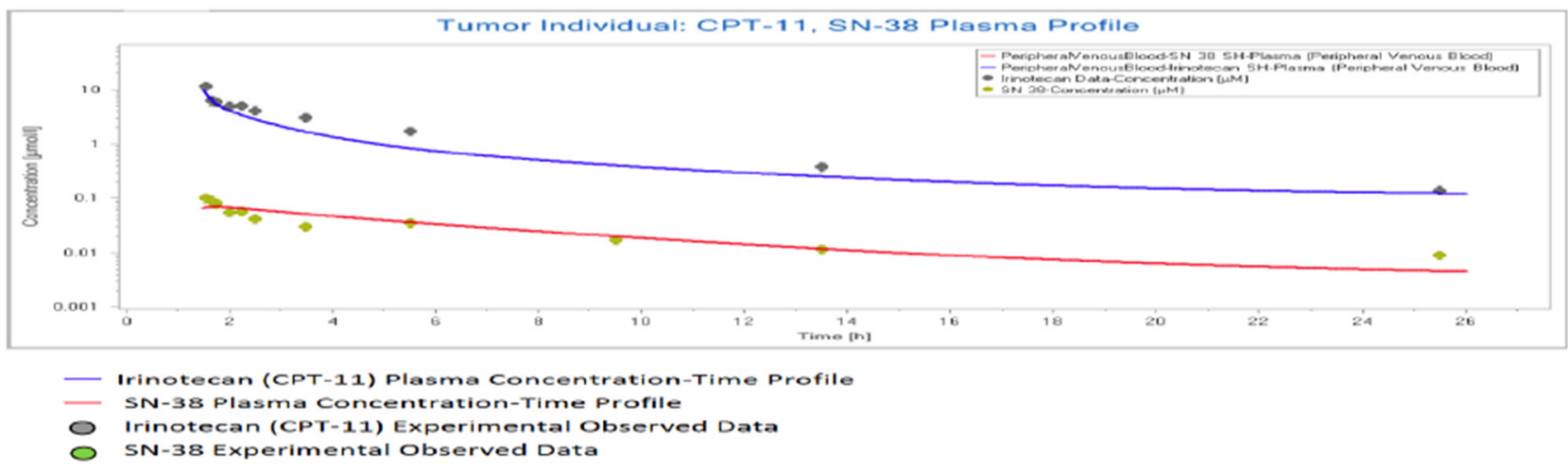

Figure 2: Population simulation result in PK-Sim. Predicted plasma profile of CPT-11 and SN-38 in an individual with tumor. 
Table 2: Physicochemical properties of CPT-11 and SN-38

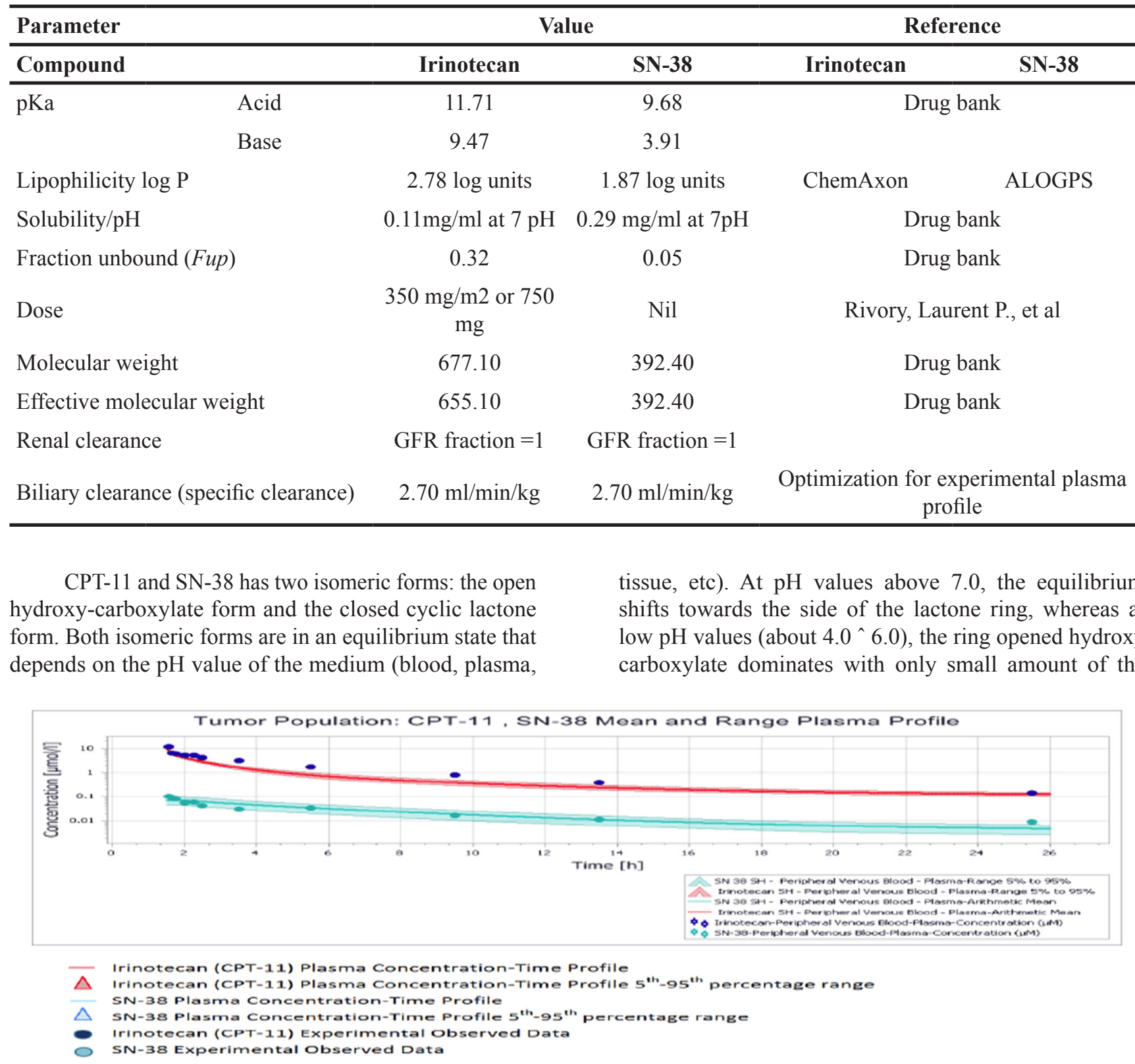

Figure 3: Mean and $5^{\text {th }}-95^{\text {th }}$ percentile plasma profile of CPT-11 and $\mathrm{SN}-38$ in the tumor population.

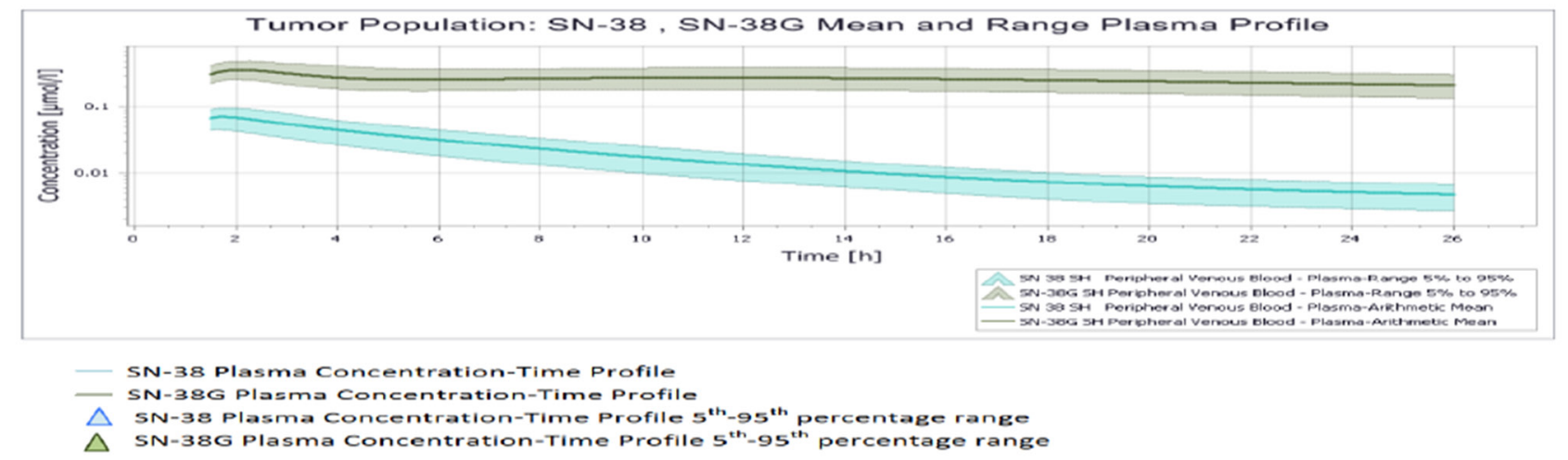

Figure 4: Mean and $5^{\text {th }}-95^{\text {th }}$ percentile plasma profile of $\mathrm{SN}-38$ and $\mathrm{SN}-38 \mathrm{G}$ in the tumor population. 
Table 3: Observed experimental data on the time (h) vs. plasma concentration ( $\mu$ mol. L) of irinotecan (CPT-11) and SN-38 after $350 \mathrm{mg} / \mathrm{m}^{2}$ irinotecan infusion

\begin{tabular}{lcc}
\hline Time (hours) & Irinotecan conc. umol/l & SN-38 conc. umol/l \\
\hline 1.56 & 11.6 & 0.1 \\
1.66 & 6.25 & 0.09 \\
1.75 & 5.95 & 0.08 \\
2 & 4.98 & 0.058 \\
2.25 & 5.05 & 0.055 \\
2.5 & 4.12 & 0.042 \\
3.5 & 3.05 & 0.03 \\
5.5 & 1.72 & 0.035 \\
9.5 & 0.8 & 0.017 \\
13.5 & 0.38 & 0.0115 \\
25.5 & 0.14 & 0.009
\end{tabular}

Table 4: Comparison of population median plasma profile and experimental plasma profile pharmacokinetics

\begin{tabular}{lccc}
\hline Parameters & $\begin{array}{c}\text { Population median CPT-11 } \\
\text { plasma conc- profile }\end{array}$ & CPT-11 experimental data & Predictive error (\%) \\
\hline AUC_tEnd $\left[\mu \mathrm{mol}^{*}\right.$ min/l] & 1296.06 & 1504.96 & 13.88 \\
Cmax $\mu \mathrm{mol} / 1$ & 9.86 & 11.6 & 15 \\
Tmax $[\mathrm{h}]$ & 1.5 & 1.56 & 3.846 \\
Half life $[\mathrm{h}]$ & 29.21 & Infinity & - \\
\hline
\end{tabular}

Table 5: Comparison of population median plasma profile and experimental plasma profile pharmacokinetics

\begin{tabular}{|c|c|c|c|}
\hline Parameters & $\begin{array}{c}\text { Population median } \mathrm{SN}-38 \\
\text { plasma conc- profile }\end{array}$ & SN-38 experimental data & Prediction error $(\%)$ \\
\hline AUC_tEnd $\left[\mu \mathrm{mol}^{*} \mathrm{~min} / \mathrm{l}\right]$ & 30.37 & 26.74 & 13.57 \\
\hline $\mathrm{Cmax} \mu \mathrm{mol} / 1$ & 0.10 & 0.10 & 0.00 \\
\hline $\operatorname{Tmax}[\mathrm{h}]$ & 1.7 & 1.56 & 8.97 \\
\hline Half life [h] & 19.47 & Infinity & - \\
\hline
\end{tabular}

lactone form. The cyclic lactone form of SN-38 represents the pharmacologic active molecule that is capable to inhibit topoisomerase while the carboxylate form is responsible for the toxic side effects of SN-38, especially diarrhea. Tumor tissue has a lower $\mathrm{pH}$ than healthy tissue or plasma hence the equilibrium shifts to the side of the hydroxy carboxylate form in tumor tissue.

However, our simulated pharmacokinetic profiles gained at different $\mathrm{pH}$ values of blood plasma and tumor tissues interprets that $\mathrm{pH}$ change in tissues or plasma does not seem to have any effect on the pharmacokinetic behavior of the CPT-11 and SN-38 though it is of significant pharmacodynamic importance.
Using this model for different races of humans can explore differences that may be present among them because of possible differences in their genetic makeup. It has been shown that CPT-11 administration is associated with hepatotoxicity, an effect that is compounded by baseline obesity. Patients with a BMI index of $>25$ were found twice as much susceptible to developing liver toxicity than patients with BMI index of 25 or lower. A growing interest in PBPK modeling techniques is their potentials of confirming these types of findings or to make predictions on drug behavior in different morbidities compounded together, conditions that are frequently encountered during clinical practice, in a relatively shorter period of time. 


\section{CONCLUSIONS}

Irinotecan is a potent anticancer agent being the first line agent in the treatment of multiple oncologic conditions. As with other chemotherapeutic agents, the severity and incidence of adverse effects limit its clinical application. As PBPK modeling has successfully been applied to assess various untested clinical situations, the model we developed to understand the behavior of irinotecan may be used to gain knowledge about potential exposure changes of this drug in cancer patients with various co-morbid conditions such as organ failure or metabolic disorders. In the future, with the availability of more and more innovative techniques for measuring tissue drug concentrations, this type of predictive tissue-based PK modeling may supervene and integrate to medical fields for delivering better treatment to patients with dose individualization according to their health status and increasing the success of chemotherapy.

\section{MATERIALS AND METHODS}

The PBPK modeling was established in computer using the software Pk-Sim 6.0 and MoBi 6.0. Initially, a virtual whole body PBPK model was developed for an adult of European race having biometrical values set according to the ones used in experimental study by Rivoryet al. [25]. A literature survey was carried out to collect the physicochemical attributes of irinotecan for molecular modeling that are shown in Table 2. Irinotecan (CPT-11) shows complex metabolism, and efforts have been put to incorporate complete metabolic and transport processes for CPT-11 and $\mathrm{SN}-38$. The metabolic conversion of CPT-11 to SN-38 is mainly through carboxyl esterase. However, CYP3A4 is also involved indirectly in this process. UGT enzyme family specifically UGT1A1, UGT1A6, and UGT1A9 further metabolize SN-38 to a more water soluble product SN-38 G. CPT-11 and SN38 movement from cell to interstitial space and through bile to intestine is carried out by various transport proteins which are incorporated in this model development.

Our Irinotecan PBPK modeling uses previously determined standard human physiological and anatomical data set for the organ volumes and blood flows. As irinotecan is eliminated through biliary route, specific biliary clearance is mentioned in the model development and kinetics of specific enzymes were incorporated in the building blocks and the simulation was created by linking together the individual's data and molecular data. The administration dose was set as $350 \mathrm{mg}$ of Irinotecan intravenous infusion run for 30 minutes according to the study reported in literature [25]. Plasma concentrationtime profile of irinotecan in this virtual individual was attained by running the simulation. This predicted profile was evaluated by applying it to the experimental data of Rivoryet al [25]. PK parameters of experimental study are shown in Table 3. Software MoBi works with PkSim in synergy and was used for tumor incorporation in this individual.

A population of 100 individuals comprising of both male and female patients was created based on the individuals' Irinotecan and SN-38 median plasma concentration-time profile. The 5 th and $95^{\text {th }}$ percentile range was obtained through population simulation using Pk-Sim. Finally, Pharmacokinetic parameters were obtained for the resultant profiles and are demonstrated in Tables 4 and 5. Furthermore, as tumor tissue has been reported to have a lower $\mathrm{pH}$ in comparison with the blood plasma, pharmacokinetic profiles of CPT-11 and SN38 were also predicted for varying $\mathrm{pH}$ ranges of tumor tissue and blood plasma in order to evaluate the effect of these changes on pharmacokinetics of CPT-11 and $\mathrm{SN}-38$. Different $\mathrm{pH}$ values between the ranges of 6.0 and 7.5 were selected for tumor tissue and blood plasma alternatively while keeping one of these at a constant value. Certain PK parameters such as AUC ${ }_{\text {t-end }}, \mathrm{C}_{\max }$, half life, plasma clearance, MRT, and volume of distribution were predicted at these $\mathrm{pH}$ environments.

\section{ACKNOWLEDGMENTS}

The authors would like to thank Bayer Technology Services, Germany for their academic support in this Modeling and Simulation work.

\section{CONFLICTS OF INTEREST}

There are no conflicts of interest.

\section{REFERENCES}

1. Jones HM, Parrott N, Jorga K, Lavé T. A novel strategy for physiologically based predictions of human pharmacokinetics. Clin Pharmacokinet. 2006; 45: 511-542.

2. Tothfalusi L, Speidl S, Endrenyi L. Exposure-response analysis reveals that clinically important toxicity difference can exist between bioequivalent carbamazepine tablets. Br J Clin Pharmacol. 2008; 65: 110-122. doi: 10.1111/j.1365-2125.2007.02984.x.

3. Kimko HC, Reele SS, Holford NH, Peck CC. Prediction of the outcome of a phase 3 clinical trial of an antischizophrenic agent (quetiapinefumarate) by simulation with a population pharmacokinetic and pharmacodynamic model. Clin Pharmacol Ther. 2000; 68: 568-577. doi: 10.1067/ mcp.2000.110975.

4. Pai MP. Drug dosing based on weight and body surface area: mathematical assumptions and limitations in obese adults. Pharmacotherapy. 2012; 32: 856-868.

5. Sawyer M, Ratain MJ. Body surface area as a determinant of pharmacokinetics and drug dosing. Invest New Drugs. 2001; 19: 171-177. 
6. U.S. Food and Drug Administration. Hematology/Oncology (Cancer) Approvals \& Safety Notifications 2014.

7. Upton RN, Mould DR. Basic concepts in population modeling, simulation, and model-based drug development: part 3-introduction to pharmacodynamic modeling methods. CPT Pharmacometrics Syst Pharmacol. 2014; 3: e88.

8. Canal P, Chatelut E, Guichard S. Practical treatment guide for dose individualisation in cancer chemotherapy. Drugs. 1998; 56: 1019-1038.

9. Evans WE, Relling MV, Rodman JH, Crom WR, Boyett JM, Pui CH. Conventional compared with individualized chemotherapy for childhood acute lymphoblastic leukemia. N Engl J Med. 1998; 338: 499-505.

10. Barrett JS, Mondick JT, Narayan M, Vijayakumar K, Vijayakumar S. Integration of modeling and simulation into hospital-based decision support systems guiding pediatric pharmacotherapy. BMC Med Inform Decis Mak. 2008; 8: 6.

11. Rowland M, Balant L, Peck C. Physiologically based pharmacokinetics in Drug Development and Regulatory Science: a workshop report (Georgetown University, Washington, DC, May 29-30, 2002). AAPS PharmSci. 2004; 6: E6.

12. Gerlowski LE, Jain RK. Physiologically based pharmacokinetic modeling: principles and applications. J Pharm Sci. 1983; 72: 1103-1127. doi: 10.1002/jps.2600721003.

13. Laplanche R, Meno-Tetang GM, Kawai R. Physiologically based pharmacokinetic (PBPK) modeling of everolimus (RAD001) in rats involving non-linear tissue uptake. J Pharmacokinet Pharmacodyn. 2007; 34: 373-400. doi: 10.1007/s10928-007-9051-7.

14. Zager MG, Schlosser PM, Tran HT. A delayed nonlinear PBPK model for genisteindosimetry in rats. Bull Math Biol. 2007; 69: 93-117. doi: 10.1007/s11538-006-9068-x.

15. U.S. Cancer Statistics Working Group. United States Cancer Statistics: 1999-2013 Incidence and Mortality Web-based Report. Atlanta (GA): Department of Health and Human Services, Centers for Disease Control and Prevention, and National Cancer Institute. 2016.

16. Slatter JG, Su P, Sams JP, Schaaf LJ, Wienkers LC. Bioactivation of the anticancer agent CPT-11 to SN-38 by human hepatic microsomal carboxylesterases and the in vitro assessment of potential drug interactions. Drug Metab Dispos. 1997; 25: 1157-1164.
17. Friedman HS, Petros WP, Friedman AH, Schaaf LJ, Kerby T, Lawyer J, Parry M, Houghton PJ, Lovell S, Rasheed K, Cloughsey T, Stewart ES, Colvin OM, et al. Irinotecan therapy in adults with recurrent or progressive malignant glioma. J Clin Oncol. 1999; 17: 1516-1525.

18. Creemers GJ, Lund B, Verweij J. Topoisomerase I inhibitors: topotecan and irinotecan. Cancer Treat Rev. 1994; 20: 73-96.

19. Innocenti F. UGT1A1 genotyping in patients undergoing treatment with irinotecan.Clin Adv Hematol Oncol. 2005; 3: 843-844.

20. Kawato Y, Aonuma M, Hirota Y, Kuga H, Sato K. Intracellular roles of SN-38, a metabolite of the camptothecin derivative CPT-11, in the antitumor effect of CPT-11. Cancer Res. 1991; 51: 4187-4191.

21. FDA Review Letter. www.accessdata.fda.gov/drugsatfda docs/appletter/1998/20571s81tr.pdf.

22. Singh S, Dwarakanath BS, Lazar MT. Role of topoisomerases in cytotoxicity induced by DNA ligand Hoechst-33342 and UV-C in a gliomas cell line. Indian J Exp Biol. 2005; 43: 313-323.

23. Wang-Gillam A, Li CP, Bodoky G, Dean A, Shan YS, Jameson G, Macarulla T, Lee KH, Cunningham D, Blanc JF, Hubner RA, Chiu CF, Schwartsmann G, et al. Nanoliposomalirinotecan with fluorouracil and folinic acid in metastatic pancreatic cancer after previous gemcitabinebased therapy (napoli-1): a global, randomised, open-label, phase 3 trial. Lancet. 2016; 387: 545-557.

24. Manallack DT. The $\mathrm{pK}(\mathrm{a})$ distribution of drugs: application to drug discovery. Perspect Medicin Chem. 2007; 1: 25-38.

25. Rivory LP, Haaz MC, Canal P, Lokiec F, Armand JP, Robert J. Pharmacokinetic interrelationships of irinotecan (CPT-11) and its three major plasma metabolites in patients enrolled in phase I/II trials. Clin Cancer Res. 1997; 3: 1261-1266.

26. Takimoto $\mathrm{CH}$. Pharmacokinetics and pharmacodynamic biomarkers in early oncology drug development. Eur J Cancer. 2009; 45: 436-438. doi: 10.1016/ S0959-8049(09)70080-2.

27. Yap TA, Sandhu SK, Workman P, de Bono JS. Envisioning the future of early anticancer drug development. Nat Rev Cancer. 2010; 10: 514-523. doi: 10.1038/nrc2870. 